\title{
LA BIOLOGIA SINTETICA COME UN NUOVO QUADRO INTERPRETATIVO DEI SISTEMI VIVENTI ${ }^{1}$
}

\author{
Nota del m.s. VÍCTOR DE LORENZO (*)
}

(Adunanza del 2 ottobre 2014)

SunTO. - La Biologia Sintetica non è solo una riformulazione contemporanea delle tecnologie del DNA ricombinante degli ultimi 30 anni combinato con un linguaggio descrittivo importato dall'ingegneria elettrica e industriale. È anche una nuova chiave interpretativa dei sistemi viventi e una dichiarazione di intenti per l'uso e la riprogrammazione degli oggetti biologici a beneficio umano. Così come la Chimica scientifica iniziata da Lavoisier ha fatto irruzione nell'Ingegneria Chimica che è alla base della nostra società sviluppata, la Biologia ha acquisito un potenziale trasformatore che probabilmente ci porterà a un tipo di industria e di economia molto diversa da quella attuale. A tal fine è essenziale identificare i colli di bottiglia che limitano il disegno di oggetti biologici da principi primi e non perdere il treno della Biologia Sintetica nella sua fase fondazionale, quando è la mente -e non il braccio- ad essere determinante.

$* * *$

ABSTRACT. - Synthetic Biology is not just a contemporary update of the recombinant DNA technologies of the past 30 years along with a descriptive language imported from electrical and industrial engineering. It is also a new interpretative key of living systems as well as a declaration of intent on the use and reprogramming of biological objects for human benefit. In the same way that scientific chemistry initiated by Lavoisier evolved into the Chemical Engineering that is the basis of our industrial society, Biology has ac-

Spagna.

E-mail: vdlorenzo@cnb.csic.es

1 Una versione estesa di questo articolo è stato pubblicato in lingua spagnola: Lorenzo, V. de (2014) Biología sintética: la ingeniería al asalto de la complejidad biológica. Arbor, 190 (768): a149. 
quired a transforming potential that will possibly lead to a type of industry and economy very different from the current paradigm. To this end, it is essential to identify bottlenecks that limit the design of biological objects from first principles and not to miss the train of Synthetic Biology at its current foundational stage, when talent -and not the muscle- is what really matters.

\section{INTRODUZIONE}

Dall'inizio del millennio la Biologia ha iniziato a sperimentare una transizione accelerata tra essere una scienza prevalentemente descrittiva ed essere una disciplina quantitativa. Questo processo è iniziato con il famoso saggio di E. Schrödinger Che cos'è la vita? scritto alla fine della Seconda Guerra Mondiale (Schrödinger, 1944) nel quale per la prima volta si propone l'approccio scrupoloso ai sistemi biologici come entità soggette alle stesse regole della Fisica del resto del mondo materiale. Il punto culminante di questa visione è stata la decifrazione 50 anni fa e nel periodo immediatamente successivo della struttura del DNA, del codice genetico e degli elementi che partecipano al flusso di informazione dal DNA alle proteine. Eppure, paradossalmente, il protagonismo dei Fisici nella nascita della Biologia Molecolare non si è tradotto in una cultura quantitativa e in un linguaggio descrittivo preciso e standardizzato caratteristico delle scienze dure. Decisamente al contrario, la Genetica Molecolare e la Biologia che da essa ha avuto origine non ha saputo approfittare (salvo contate eccezioni) dell'opportunità di formalizzare i meccanismi e le funzioni presenti nei sistemi viventi con enunciati e codici precisi. Ne sono susseguiti decenni di completo scompiglio nella nomenclatura dei geni e nella forma di misurare e quantificare le attività biologiche, per non parlare dell'esplosione di ogni tipo di vettori di DNA per la manipolazione genetica dei sistemi sperimentali studiati. Forse l'booliganismo scientifico glorificato nella Doppia elica di J. Watson (Watson, 1968) non è stato estraneo all'ethos informale e antiautoritario della comunità scientifica nata dopo quel periodo. Mentre questo non è stato un problema per molto tempo, è indiscutibile che sia i progressi propri in questo ambito sia il ruolo crescente della Biologia e della Biotecnologia in campi al di là del contesto accademico tornano a reclamare con forza la necessità di dotare le Scienze della Vita di metodologie e linguaggi più vicini alla Fisica che alle scienze narrative - così com'è stata la Biologia per gran parte della sua storia. $\grave{\mathrm{E}}$ in questo contesto che si manifestano i due ultimi tentativi di quanti- 
ficare la Biologia, che sono chiamati a cambiare per intero - sia metodologicamente che concettualmente, i nostri approcci alle domande scientifiche e ai loro derivati biotecnologici.

\section{DALla Biologia MOLECOlaRE ALLA BiOlOGIA DEI SISTEMI E ALLA BIOLOGIA SINTETICA}

L'inizio della Biologia dei Sistemi è stato determinato da un problema molto pratico: come organizzare e come trovare un senso alla valanga di dati derivati dalle tecnologie omiche che dalla fine degli anni ' 90 si sono iniziate ad applicare ai sistemi biologici. Al sequenziamento del genoma completo hanno poi fatto seguito i trascrittomi, i proteomi e i metabolomi. A questi sono successi in maniera naturale le loro versioni meta corrispondenti (cioè, dati di popolazioni multispecie), così come l'esame degli stessi dati in cellule individuali. Sfortunatamente, $i$ dati per se non si trasformano automaticamente in informazione e ancora meno in conoscenza. Per questo è necessario processarli con tecnologie che non provengono in assoluto dalla Biologia, ma dalla computazione, dall'informatica e dalla fisica di sistemi complessi. Infatti, le tecniche omiche permettono di disporre di tutti i dati contenuti in un sistema biologico, ma in una forma più o meno criptica che ha bisogno di essere decifrata per essere compresa, ma con strumenti estranei alla Biologia (ad esempio la teoria delle reti; Barabasi e Oltvai, 2004). Questo implica al tempo stesso un'opportunità (capire il sistema vivente per intero più che le sue parti separatamente) e una grande sfida metodologica ed epistemologica. Da una parte, l'analisi dei dati massivi si trasforma non solo in un aiuto alla sperimentazione, ma anche in una fonte genuina di nuova informazione e conoscenza. Questa forma di investigare è estranea alla grande tradizione ipotetico-deduttiva della Biologia sperimentale, ma è molto probabile che sia ugualmente valida. Branche intere della Biologia che 25 anni fa erano completamente sperimentali (ad esempio, l'Ecologia Microbica) si stanno trasformando in grandi piattaforme di analisi di sequenze. Dall'altra parte, i dati devono proiettarsi sempre su qualche modello di funzionamento, e questo ha portato una buona parte dei Biologi Sistemici a specializzarsi nella rappresentazione di dati e nella modellazione del loro funzionamento, spesso importando formalismi dalle reti sociali e dai circuiti elettronici. Questo a sua volta genera nuove domande e nuovi filoni di ricerca, il 
cui obiettivo è comprendere la complessità multiscala degli oggetti viventi. Per la prima volta si considera la possibilità di capire l'architettura materiale (l'hardware) e la logica operativa (il software) di un sistema vivente (Danchin, 2009a). E per capire il tutto è necessario studiare il tutto come tale, non concentrarsi soltanto nei dettagli dei suoi componenti. Con questo fine è necessario utilizzare astrazioni e semplificazioni tipiche della Fisica che aiutino a separare i componenti principali di un sistema da quelli che soltanto sono spettatori o di passaggio.

Questo porta alla tappa successiva: la definizione dei componenti minimi che deve avere un sistema biologico per mantenere la sua identità e le sue funzioni. E alla fine, la comprensione totale di un sistema richiede non solo la sua analisi, ma anche la sua sintesi, così come proclamava nella sua celebre lavagna postuma il Premio Nobel di Fisica Richard Feynman "...quello che non posso creare, non lo capisco...”. È pertanto la Biologia dei Sistemi e la sua enfasi nella quantificazione, la modellizzazione e l'uso combinato dell'analisi e la sintesi per capire le entità viventi che all'inizio del millennio ha preparato lo scenario per la nascita e lo sviluppo esplosivo della Biologia Sintetica, così come lo stiamo vivendo oggigiorno.

\section{GUARDARE I SISTEMI VIVENTI CON OCCHI DA INGEGNERE}

La quantificazione della Biologia e le astrazioni che sono il segno distintivo della Biologia dei Sistemi rendono quasi inevitabile un nuovo quadro interpretativo degli oggetti viventi. La Biologia del XX secolo ha usato due chiavi ermeneutiche collegate tra loro per la comprensione dei sistemi biologici. La prima e più importante è la teoria dell'evoluzione. Così come dichiara la nota affermazione di Dobzhansky "...Nulla ba senso in Biologia, se non alla luce dell'evoluzione...". Questo chiarisce la ragion d'essere degli oggetti biologici come risultato di un processo temporale non-orientato di complessità e di interattività a beneficio dell'adattamento ambientale e del successo riproduttivo. L'altra chiave è ciò che è stato denominato il Dogma Centrale (DC) della Biologia Molecolare, cioè il flusso dell'informazione DNA $\rightarrow \mathrm{RNA} \rightarrow$ Proteine. Queste due chiavi (l'evoluzione e il DC) permettono di rispondere alla domanda sul perché $i$ sistemi biologici sono così come sono e come li conosciamo. Ma, in realtà, le stesse chiavi non ci dicono gran cosa sul funzionamento del sistema naturale stesso, e molto meno sulla possibilità che questo possa 
essere distinto, meccanicisticamente, rispetto a ciò che vediamo qui e ora.

L'evoluzione seleziona le funzioni e le sue costellazioni, ma non necessariamente i meccanismi specifici che proporzionano tale funzionalità. Questo si può vedere chiaramente nel mondo procariotico: uno stesso problema metabolico o regolatore può risolversi attraverso schemi molecolari distinti (Cases e de Lorenzo, 2001). La domanda su come funziona un sistema biologico è pertanto difficile da rispondere con una mera ottica evolutiva. E qui giunge la proposta rivoluzionaria della Biologia Sintetica: per capire il funzionamento dei sistemi viventi dobbiamo contemplarli come se fossero oggetti non distinti da quelli che disegna un ingegnere computazionale, chimico o elettronico (Endy, 2005). Ad esempio, per capire la distribuzione spazio-temporale del metabolismo in un batterio non ci è di grande aiuto conoscere la sua origine evolutiva. Al contrario, ci dovremmo interrogare su cosa si chiederebbe un ingegnere chimico per creare un microreattore nel quale avvengono migliaia di reazioni simultaneamente nello spazio e nel tempo. Da questa domanda si deduce, ad esempio, la necessità di compartimentalizzazione (o almeno di limitazione della libera diffusione), l'esistenza di canalizzazione di substrati e prodotti, l'invecchiamento delle proteine, il problema dei residui tossici, ecc. (de Lorenzo e Danchin, 2008). Solo con questa prospettiva ingegneristica possiamo capire quel sistema fisico-chimico che è la cellula nello spazio e nel tempo - lasciando da parte la domanda sulle sue origini.

In generale, gli oggetti procedenti dall'ingegneria devono compiere una funzione per la quale hanno bisogno di alcune istruzioni (il software nei computer) che si implementano attraverso un hardware (la macchina che legge ed esegue le istruzioni). La Biologia Molecolare tradizionale tende a dimenticare la distinzione tra funzione, istruzioni (software e sistema operativo) e il macchinario per eseguirle (hardware), che richiede una certa rivisitazione della frase di Dobzhansky prima citata: l'evoluzione seleziona prevalentemente le funzioni e le sue combinazioni, ma non tanto le istruzioni né le forme di leggerle. Ciò che sì è caratteristico dei sistemi viventi è che buona parte del loro software/hardware è dedicato alla loro autoreplicazione. In questo senso, così come ha proposto $\mathrm{A}$. Danchin, le cellule possono somigliare a macchine di Turing capaci di interpretare simboli (ad esempio chimici: ATGC) contenuti in un nastro (il DNA/RNA nel nostro caso) conforme a una tabella di regole nello stesso modo in cui lo fanno i computer (Danchin, 2009b). Una macchina di questo tipo può essere adattata per 
eseguire ogni tipo di operazione, comprese le istruzioni sul proprio assemblaggio. Portando questa metafora all'estremo, le cellule possono intendersi come computer che fanno computer (Danchin, 2009a).

\section{TECNO-LOGIA VS TECNO-NOMIA}

Tuttavia, l'enunciato Biologia-come-ingegneria richiede varie precisazioni. In primo luogo, il guardare gli oggetti biologici come se fossero il prodotto di una ingegneria non dice nulla sull'intervento di un ingegnere. Un argomento simile è stato usato da J. Monod nella sua encomiata discussione sulla teleologia (finalità dei sistemi biologici) e la teleonomia (apparenza di finalità nei sistemi stessi) del suo libro Il caso e la necessità (Monod, 1970). Mentre la prima non è accreditata come fatto scientifico, la seconda è una chiave interpretativa estremamente utile per capire perché i sistemi biologici sono come sono invece di essere diversi. Per lo stesso motivo, si può adattare l'ingegneria a una metafora e a una chiave ermeneutica per capire la logica degli oggetti biologici, che è distinta, ma perfettamente compatibile con altre chiavi che affrontano domande diverse. In questo senso, alla tensione teleologia (finalità) vs. teleonomia (apparenza della finalità) corrisponderebbe una correlazione tecno-logia (disegno)/tecno-nomia (apparenza di disegno).

Tralasciando questi argomenti piuttosto speculativi, possiamo realmente considerare i sistemi viventi con la prospettiva di un ingegnere? Ai suoi tempi, F. Jacob propose un contrasto insuperabile tra l'ingegneria e il bricolage come metafora di quanto sia distinto il disegno razionale dall'evoluzione biologica (Jacob, 1977). Mentre il lavoro degli ingegneri si basa su componenti precisi e strumenti che si adeguano esattamente a un progetto predefinito, l'amante del bricolage gioca con cianfrusaglie e rimasugli senza sapere ciò che produrrà e usa tutto ciò che trova per creare qualsiasi tipo di oggetto funzionale, la cui utilità potrebbe manifestarsi in un secondo momento: nessuno dei materiali possiede all'inizio una funzione precisa e ognuno si può utilizzare in maniera diversa. Tuttavia, questa visione sembra trasmettere che la struttura dei sistemi viventi non ha nessuna logica relazionale comparabile all'ingegneria. Niente di più lontano dalla realtà. Nonostante le soluzioni a problemi di disegno possano presentarsi su strade diverse, le due con frequenza coincidono o convergono (ad esempio, forme di aerei $v s$ uccelli) e una può facilmente aiutare a capire l'altra. 
Lo stesso può applicarsi ai sistemi biologici: anche se la loro struttura e funzionamento non possono attribuirsi ad un ingegnere, è molto utile esaminarle con la prospettiva e i formalismi che proporziona l'ingegneria. Funzioni e moduli biologici che hanno rappresentato un'innovazione evolutiva per risolvere un problema hanno avuto il loro maggior successo quando sono stati poi applicati in un altro contesto per raggiungere un obiettivo diverso. Ad esempio, il piumaggio apparve a suo tempo come un mero isolamento termico ma poi si è trasformato in un componente essenziale del volo degli uccelli. L'analisi dei genomi batterici proporziona innumerevoli esempi di proteine che nascono per fare qualcosa che poi risulta essere molto diverso da quello per cui erano sorte in un primo momento (Milanesio et al., 2011). Questo processo, che in Biologia evolutiva si denomina esaptazione, ha anche innumerevoli controparti in ingegneria: un dispositivo che si inventa con un proposito molto specifico riappare con piccole modifiche in un altro luogo con una finalità inaspettata. Il sistema di carica e sgancio rapido di bombe negli aerei da combattimento si riutilizza per l'incorporazione e scarica di pesanti batterie nelle macchine elettriche. Uno stesso dispositivo si inventa per una funzione ma alla fine si afferma quando gli si assegna un'altra distinta e addirittura opposta a quella iniziale. Questo scenario è costantemente presente nei sistemi biologici e nei sistemi disegnati e permette di ridurre la frattura insuperabile tra i due, così come lo aveva prospettato Jacob. Tuttavia, il principio di tecno-nomia che si propone in questo articolo sarà difficile da discutere serenamente in un momento in cui il dibattito tra l'evoluzionismo e il disegno intelligente si è diffuso in maniera virale nei paesi anglosassoni e si è riverberato su tutti gli altri. In ogni caso e in modo più o meno esplicito, guardare gli oggetti biologici con occhi da ingegnere è e sarà il più grande $\mathrm{e}$ utile pilastro concettuale della Biologia Sintetica.

\section{La modularità dei sistemi biologici}

Una seconda motivazione sulla relazione Biologia-Ingegneria ha a che vedere con la struttura modulare degli oggetti di studio in ogni caso. Qualsiasi entità disegnata da un ingegnere è composta da moduli chiaramente definiti, con connessioni tra i suoi componenti ben standardizzate (che permette il suo riutilizzo in contesti differenti), con entrate e uscite compatibili e una chiara gerarchia e disposizione in tre dimensioni dei distinti componenti. Questo fa sì che la modularità fisica degli ogget- 
ti fatti dagli ingegneri coincida, almeno approssimativamente, con quella funzionale. Al contrario, i sistemi biologici esistenti non sembrano manifestare a prima vista questa coincidenza tra il fisico e il funzionale. Il catalogo di funzioni necessarie per dare vita a un sistema si è potuto quantificare in circa 300-500, paragonando gruppi di geni persistenti nei genomi microbici (de Lorenzo e Danchin, 2008). Tuttavia, cercando geni specifici condivisi tra quei stessi genomi, la conclusione sorprendente è che quel numero è esattamente zero (Acevedo-Rocha et al., 2013). Questo significa che le stesse necessità funzionali possono essere coperte da configurazioni molto diverse di geni e molecole.

Un altro particolare degno di nota che separa gli oggetti disegnati e i sistemi biologici sono le caratteristiche fisiche dei loro componenti: i telefoni e gli aerei sono fatti con materiali duri, con componenti perfettamente definiti nella loro struttura tridimensionale e con connessioni precise con i componenti vicini. La comparsa di interazioni non previste in genere dà problemi e causa incidenti. Al contrario, oggetti biologici sono tipicamente composti da elementi labili, a volte senza limiti ben chiari e con la tendenza a interagire gli uni con gli altri - ciò che a volte porta alla comparsa di proprietà emergenti non anticipate. Se l'ingegneria elettrica e industriale si occupa di cavi, tubi e viti, i sistemi viventi sono composti da elastomeri, gelatine e colle. In ultimo, i sistemi viventi crescono, replicano e si riproducono - proprietà estranee agli oggetti fatti dall'ingegneria razionale che conosciamo. Questo può significare che il principio di modularità che associamo ai dispositivi fatti dall'uomo è assente nei sistemi biologici? Ancora una volta la risposta è no. La complessità delle cellule con grandi genomi ed estesa diversità biochimica è ingannevole a questo rispetto. L'analisi dei genomi minimi trovati, ad esempio nei batteri endosimbionti, rivela un considerevole grado di modularità nelle funzioni essenziali che permettono la loro esistenza (Porcar et al., 2013). La zuppa biochimica che a volte il metabolismo sembra essere è perfettamente modularizzata, con un'organizzazione che ricorda un'industria chimica. Nella direzione contraria, nemmeno l'idea di oggetti auto-replicanti è nuova in ingegneria, così come dimostrano nell'ultimo decennio i tentativi di disegnare stampanti tridimensionali che stampino se stesse (ad esempio il progetto RepRap: http://reprap.org/).

È pertanto possibile e produttivo usare la metafora e anche i formalisimi dell'ingegneria per capire il funzionamento dei sistemi biologici come la metafora biologica per indirizzare il disegno di nuovi 
dispositivi creati dall'uomo. In questo senso, una buona parte dell'ingegneria contemporanea è abituata a investigare aleatoriamente lo spazio di soluzioni a un problema la cui risposta non si può intuire sin dall'inizio. Per questo, si adottano strategie di prova ed errore che non sono molto distinti dai processi evolutivi in Biologia. Lo stesso Gaudí, in un momento storico senza capacità computazionale né simulazioni che sono oggi così abituali nell'architettura moderna, à stato capace di calcolare parametri complessi nei suoi edifici a forza di interrogare la natura (nel suo caso, per pura gravità in modelli di corde e pesi) sulla configurazione ottimale dei componenti delle sue grandi opere. Ciò che più interessa in questo caso è che tali soluzioni siano virtualmente identiche a quelle trovate dagli architetti molti anni dopo usando calcoli e simulazioni avanzate (Huerta, 2006). Sembra pertanto che nell'ingegneria come nella Biologia, lo spazio di soluzioni a un obiettivo adattativo abbia attrattori nei quali la soluzione stessa può essere il risultato di un disegno guidato o una esplorazione aleatoria. A effetti pratici, questo accredita il valore e l'utilità di esaminare la logica composizionale dei sistemi viventi con gli strumenti concettuali dell'ingegneria.

\section{L'ingegneria genetica: analogia e metodologia}

L'opzione dell'ingegneria come chiave per interpretare fenomeni biologici che costituiscono la Biologia Sintetica implica una conseguenza affascinante e allo stesso tempo inquietante. Non è solo una questione epistemologica, ma anche molto pratica. Se un sistema biologico è come un artefatto realizzato dall'ingegneria, allora possiamo anche scomporlo in un insieme limitato di componenti definiti che poi possiamo ricomporre per generare un oggetto distinto seguendo un piano razionale. Il risultato può essere un oggetto con una struttura e delle proprietà distinte da quelle che aveva la fonte originale di quei stessi componenti. Ma per farlo abbiamo bisogno di due cose. In primo luogo, l'astrazione relazionale e gerarchica del nuovo oggetto come un insieme di parti (le unità basiche di funzione biologica) che si collegano in maniera razionale per formare dispositivi e questi a loro volta interagiscono per generare sistemi di complessità crescente. A questo punto saltiamo dall'ingegneria come metafora e analogia (come nell'ingegneria genetica) all'ingegneria come autentica metodologia per costruire oggetti biologici. In questo senso, la formulazione narrativa della Biologia Molecolare come un processo DNA $\rightarrow$ RNA $\rightarrow$ proteine viene sostituita 
dal principio composizionale e quantitativo della Biologia Sintetica: Parti $\rightarrow$ dispositivi $\rightarrow$ sistemi (Fig. 1).
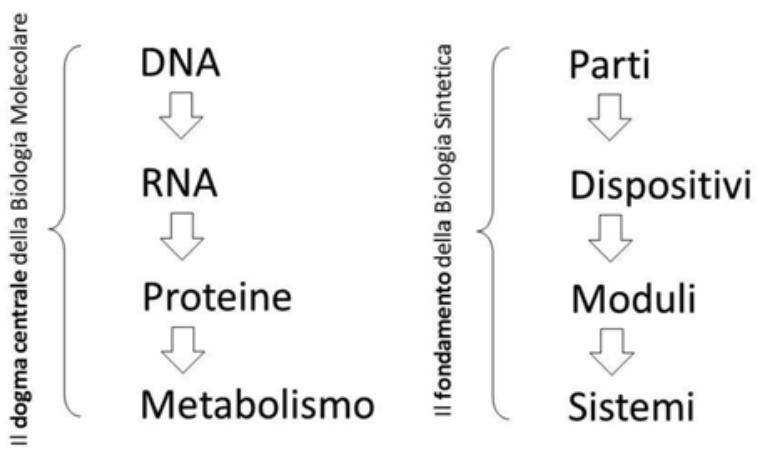

Il dogma centrale della Biologia Molecolare (sinistra) si basa nel trasferimento di informazione dai nucleotidi del DNA alle proteine e (come è stato proposto recentemente: de Lorenzo, 2014) la propagazione di questo flusso di informazione sul funzionamento delle reti metaboliche. Al contrario, la Biologia Sintetica (destra) mette in rilievo la logica relazionale e composizionale dei sistemi viventi, sia quelli esistenti sia quelli che si potranno disegnare in futuro.

Fig 1. - La Biologia Molecolare e la Biologia Sintetica affrontano i sistemi viventi con diverse chiavi interpretative.

In secondo luogo, le parti per l'ingegneria di nuovi sistemi biologici hanno bisogno di essere standardizzate per renderle riutilizzabili, componibili e scalabili. Nella maggior parte dei casi, queste parti non appaiono così nella loro situazione naturale. Possiamo fare una capanna con i tronchi di alberi così come si trovano in Natura. Ma per fare una casa i tronchi devono essere trasformati in travi e pannelli di dimensioni precise per permettere la costruzione di un edificio più complesso. Mediante questo ragionamento, uno dei segni distintivi della Biologia Sintetica è lo sforzo di partire da sequenze di DNA che determinano funzioni d'interesse e modificarle affinché si possano utilizzare come blocchi di costruzione (biomattoni) di nuovi oggetti biologici (Kosuri et al., 2013; Mutalik et al., 2013). Partendo dalla situazione esistente, si può pensare di modulare sempre più le funzioni e i componenti biologici per renderli più facili da combinare, sia fisicamente che funzionalmente. Quest'obiettivo di modularizzazione e standardizzazione apre prospettive immense alla Biotecnologia: i sistemi viventi si trasformano 
in una fonte di materiali che permettono di generare nuovi oggetti e proprietà poco o per niente simili alla loro funzione naturale. Un promotore batterico, che nel suo contesto nativo controlla l'espressione di un gene di resistenza a tetraciclina, quando le cellule trovano l'antibiotico nel terreno di coltura, si trasforma per opera e arte della Biologia Sintetica in un modulo inversore (una porta logica NOT) che può combinarsi con altre per eseguire calcoli e processare segnali che in origine non sono i suoi (Silva-Rocha e de Lorenzo, 2008). Distinti enzimi batterici e vegetali possono integrarsi in un lievito per dar vita a una via biosintetica di una droga anti-malarica (Paddon e Keasling, 2014). Luoghi di ancoraggio di proteine derivate da vie di segnaletica di metazoi sono stati utilizzati per canalizzare in Escherichia coli i substrati di una biotrasformazione di interesse industriale (Dueber et al., 2009). E così decine di casi nei quali una funzione biologica si decontestualizza con gli strumenti del DNA ricombinante (e più recentemente attraverso sintesi chimica di sequenze di DNA) e si riutilizza in una situazione distinta per fare qualcosa che la natura non ha fatto o inventato prima.

\section{SEMPLIFICARE LA Biologia PER RENDERLA PIÙ FACILE} DA (RI)DISEGNARE

Questo anelito collide con due grandi obiettivi. Da una parte, la composizione fisica delle sequenze di DNA non si traduce necessariamente in una integrazione delle funzioni corrispondenti, almeno in forma quantitativa. E i parametri associati agli elementi biologici (ad esempio, promotori, terminatori, luoghi di unione di ribosomi, ecc.) cambiano spesso con il contesto genomico e con le condizioni fisiologiche dell'ospite. Infatti, il problema della dipendenza di contesto è una delle maggiori limitazioni per disegnare dispositivi biologici affidabili. Per rimediare questo stato delle cose sono state proposte varie strategie.

Una di queste è riesaminare il genoma per eliminare la complessità che non sia strettamente necessaria per una certa applicazione. In una prima fase, il genoma si può pulire da elementi che causano instabilità (ad esempio, profagi, sequenze di inserzione, elementi mobili, ecc.), per continuare poi con blocchi di geni che, nonostante siano presenti e utili nell'ambiente naturale, possono non essere essenziali (ad esempio, il macchinario flagellare) in un bioreattore (Posfai et al., 2006). Poi, si potrebbero eliminare i blocchi metabolici non utilizzati, strutture del- 
l'involucro cellulare e molti altri geni che possono considerarsi non necessari. Nonostante con questo approccio si potrebbe minimizzare il genoma e pertanto il contesto molecolare di qualsiasi dispositivo che si potrebbe impiantare in esso, di fatto i tentativi di ridurre il genoma di batteri modello come E. coli non sono stati in grado di andare oltre il 2030\% (Posfai et al., 2006). A parte l'eliminazione di possibili geni essenziali, la delezione di grandi segmenti del cromosoma potrebbe alterare la sua architettura dentro la cellula e renderlo non funzionale. Un'alternativa sarebbe andare nella direzione esattamente contraria: partire da batteri con genomi già molto piccoli, ad esempio Mycoplasma o endosimbionti come Buchnera (van Ham et al., 2003). In questi casi, la natura stessa ha fatto il lavoro di riduzione. Anche se in principio questo potrebbe essere un buon approccio, che un sistema abbia meno componenti non significa che la situazione sia più semplice. La riduzione di complessità composizionale si compensa con l'incremento della complessità relazionale: i cromosomi con meno geni danno vita a cellule che sono molto più dipendenti alle loro interazioni con l'ambiente. Nonostante questo, alcuni batteri con genomi piccoli (come Mycoplasma) sono cavalli di battaglia di riferimento in Biologia Sintetica. Soprattutto perché la dimensione del cromosoma permette di realizzare la sua sintesi chimica completa, così come l'aveva effettuata il gruppo di Venter (Gibson et al., 2010). Questo apre la possibilità di mettere in pratica lo scenario sopra citato, ossia di considerare i batteri e altri sistemi biologici come computer per i quali si può scrivere un software (il DNA) che può essere eseguito da un macchinario molecolare già esistente. Le proposte futuriste di Craig Venter su un futuro convertitore biologico digitale (http://www.theguardian.com/science/2013/oct/13/craig-ventnermars) vanno esattamente in questa direzione.

\section{Ortogonalizzazione}

Tuttavia, semplificare il genoma e persino riscriverlo non risolve tutti i problemi. Come si è sopra citato, il funzionamento, soprattutto quantitativo delle parti biologiche, è soggetto in maggior o minor misura all'influenza di vari livelli di contesto - da interferenze con sequenze prossime a effetti globali e medio ambientali. A beneficio dell'evoluzione ma a scapito dei bioingegneri, i materiali biologici (proteine, polimeri, piccole molecole, ecc.) tendono a interagire con i loro vicini molecolari in modo spesso imprevedibile. In Biologia, $2+2$ non fa sempre 4 per- 
ché ogni nuova combinazione è soggetta alla comparsa di proprietà emergenti (negative o positive) che non si possono predire a partire dalle qualità dei componenti della somma, almeno con il grado di conoscenza che abbiamo nella maggior parte dei casi. Una situazione familiare a qualsiasi biotecnologo è l'incertezza sull'efficienza di sistemi di espressione eterologa di geni di interesse industriale. La combinazione di un promotore forte con un segnale di inizio della traduzione anch'esso forte dovrebbe in linea di principio condurre a un'espressione (combinazione trascrizione+traduzione) anch'essa forte del gene di interesse. Questo risulta essere così in molti casi, ma in altri succede esattamente il contrario. Perché? Molto spesso, l'estremo 5' del trascritto forma strutture secondarie inaspettate con sequenze più in basso del gene in questione, che genera una grande instabilità nell'mRNA o impedisce la traduzione.

Un possibile rimedio a queste situazioni è la cosiddetta ortogonalizzazione dei componenti che vanno in un sistema. Due sistemi sono reciprocamente ortogonali se non si influenzano l'uno all'altro. È ragionevole partire da un componente o un modulo biologico molto connesso, produrre una variante che conservi solo la connessione desiderata e rendere così più facile il suo uso in nuovi disegni biologici. La natura stessa offre casi di parti ortogonali, solitamente in elementi mobili promiscui e batteriofagi (ad esempio, l'RNA polimerasi del fago T7). Inoltre, si sono fatti grandi passi avanti anche nell'elaborazione di codici genetici alternativi e ribosomi ortogonali capaci di decifrarli. Forse in un futuro non molto lontano potremo disporre di entità biologiche con un genoma che possa codificare l'informazione con un cifrato genetico distinto (anche con basi non naturali; Malyshev et al., 2014) che si possa trascrivere con polimerasi alternative e produca un messaggio che è traducibile mediante ribosomi ortogonali. Infatti, l'essere vivente risultante sarebbe così lontano da quello esistente che non potrebbe interagire in nessun modo con i sistemi biologici naturali, assicurando così il suo contenimento e la sicurezza del suo uso biotecnologico (Schmidt e de Lorenzo, 2012).

\section{Frenare l'evoluzione?}

Le sfide che deve affrontare la Biologia Sintetica per trasformarsi in un'autentica branca dell'ingegneria non finiscono con ciò che si è detto finora. Infatti, rimane ancora la sfida più importante: assicurarsi che qualsiasi dispositivo/essere vivente disegnato da bioingegneri man- 
tenga le sue proprietà nel tempo e non soccomba al rumore e alle mutazioni - o sviluppi proprietà inaspettate. Anche se ottenessimo un disegno ottimizzato di un circuito biologico o di un sistema completo, sarebbe comunque inevitabile che dopo un certo periodo di tempo il DNA che lo determina muti (molto di più in caso di stress ambientale) e che questo porti al collasso finale dell'entità. La letteratura scientifica e biotecnologica contiene numerosi esempi di microrganismi ricombinanti disegnati per una determinata funzione che, dopo un certo periodo di tempo, smettono di eseguire il programma genetico atteso a causa dell'accumulo di mutazioni. La domanda ovvia è se possiamo forzare in modo stabile i sistemi naturali a fare qualcosa a nostro stesso beneficio che abitualmente non fanno. La strategia predominante per affrontare questa grande sfida è quella di penalizzare (sempre attraverso circuiti genetici ad boc) le mutazioni che portano a non raggiungere gli obiettivi, ad esempio inducendo al suicidio i mutanti indesiderati. Tuttavia, come qualsiasi costruzione genetica, i circuiti di letalità condizionale sono soggetti ad acquisire mutazioni che li rendono inefficienti. Questo è un obiettivo che è stato proposto ma che è ancora assolutamente irrisolto. Le proposte passano dalla permuta delle molecole che portano l'informazione (dal DNA a polimeri parzialmente o totalmente artificiali) fino al cambio totale del supporto dell'informazione, da essere incriptata in un codice ad essere determinata da una composizione lipidica. Questo è un collo di bottiglia reale che dovrà essere affrontato perché la Biologia Sintetica arrivi a realizzare tutte le sue promesse.

\section{COSA FARE?}

L'agenda di ricerca della Biologia Sintetica si può facilmente dedurre da quanto è stato detto finora in quest'articolo. L'ostacolo oggettivo più grande per l'ingegneria di sistemi biologici è l'influenza del contesto multiscala nel funzionamento dei componenti individuali di qualsiasi entità vivente. La riduzione della complessità genomica, l'ortogonalizzazione delle parti e dei dispositivi da combinare e l'eliminazione di mutanti che perdono il proprio programma impiantato sono ovviamente strade da seguire. Ma questo non è tutto. Ci sono domande biologiche fondamentali a cui dobbiamo rispondere per poter andare avanti nel disegno di tali oggetti. La prima è chiarire la relazione tra metabolismo, crescita, divisione e proliferazione di cellule, iniziando 
dagli esseri più semplici, i batteri. Come è stato sopra citato, a differenza dei dispositivi fatti dall'uomo, i sistemi viventi crescono e questo aggiunge una complessità straordinaria nel momento di disegnarli in modo predicibile. Nel migliore dei casi, si vorrebbe disporre di cellule che eseguono le funzioni per le quali sono state disegnate ma senza crescere. Questo suppone obiettivi di ricerca affascinanti, perché qualsiasi programma implementato con componenti materiali invecchia e commette errori. E la forma che i sistemi biologici hanno per ripararli è creandoli di nuovo nelle copie che si generano durante la crescita. Dato che il fine evolutivo degli esseri viventi è il successo riproduttivo, possiamo separare quella crescita dal resto della funzionalità biologica senza alterare seriamente le cellule corrispondenti? Qui c'è un problema che difficilmente può risolvere solo la Biologia Molecolare, perché ha derivazioni nella teoria dell'informazione, nella resistenza di nanomateriali e nell'ingegneria di sistemi meccanici.

Un'altra limitazione ancora da studiare nel dettaglio è l'influenza del metabolismo nel flusso di espressione genetica. Le astrazioni dei circuiti e moduli da impiantare nei sistemi biologici spesso omettono che la loro azione si esegue in un ambiente chimico complesso con una logica propria e un'alta reattività. Quello che nel gergo della Biologia Sintetica viene denominato chassis si compone non solo di genoma più o meno intricato ma anche con una grande complessità molecolare che conosciamo appena. Da come potremo capire questa complessità multiscala e multimolecolare dipenderà in gran misura che gli sviluppi in corso della Biologia Sintetica possano trasformarsi in tecnologie robuste o restare meri aneddoti.

Nel migliore dei casi, una volta che si possiedono componenti affidabili per la costruzione di un oggetto complesso (come un Meccano o un Lego), è questione dell'immaginazione dell'utente il produrre ogni tipo di articolo e materiale, da biocombustibili, biocatalizzatori e nuovi agenti terapeutici con forme di computazione distinte, bioplastiche e fibre intelligenti o biosensori per molecole di ogni tipo. Ed è giustamente nell'immaginazione e creatività che la nostra comunità scientifica e tecnica può dare i maggiori contributi al campo e giungere laddove non sono riuscite ad arrivare le grandi iniziative in Biologia Sintetica negli Stati Uniti basate nel dominio tecnico, come il Programma SynBERC (http://www.synberc.org). Per questo è necessario fomentare l'intercambio Scienze della Vita-Ingegneria nei programmi accademici delle Università e promuovere corsi di studio che inte- 
grino in parti uguali Biologia Fondamentale e principi di Ingegneria. Per il momento, molti Biologi Molecolari adducono che gli Ingegneri sanno poco o niente di Biologia e pertanto è difficile interagire con loro. E a loro volta, gli Ingegneri ritengono che buona parte dei Biologi sia carente di talento quantitativo e di formazione matematica considerati importanti per il disegno di sistemi che funzionino veramente. Il superamento di questa barriera culturale è probabilmente il più grande obiettivo che dobbiamo affrontare, dal quale tuttavia dipendiamo per non essere meri spettatori, bensì attori del nuovo tipo di Bioindustria che vedremo svilupparsi nei prossimi decenni.

\section{RINGRAZIAMENTI}

Il progetto è stato finanziato dall'Unione Europea (Grants ARYSIS, ST-FLOW ed EVOPROG), dal Prgramma BIO del Ministero dell'Economia spagnolo e dal Progetto della Regione Autonoma di Madrid. VdL dà atto dei dibattiti con Antoine Danchin per il modo in cui illustra la sua visione della Biologia Sintetica discussa nel presente articolo.

L'autore ringrazia sinceramente la traduttrice Maria Elena Casasole per la professionalità con cui ha svolto il suo lavoro.

\section{BIBLIOGRAFIA}

Acevedo-Rocha, C.G., Fang, G., Schmidt, M., Ussery, D.W. y Danchin, A. (2013) From essential to persistent genes: a functional approach to constructing synthetic life. Trends Genet 29: 273-279.

Barabasi, A.-L. y Oltvai, Z. N. (2004) Network biology: understanding the cell's functional organization. Nat Rev Genet 5, 101-113.

Cases, I. y de Lorenzo, V. (2001) The black cat/white cat principle of signal integration in bacterial promoters. EMBO J 20, 1-11.

Danchin, A. (2009a) Bacteria as computers making computers. FEMS Microbiol Revs 33, 3-26.

Danchin, A. (2009b) Information of the chassis y information of the program in synthetic cells. Syst Synth Biol 3, 125-134.

de Lorenzo, V. (2014) From the selfish gene to selfish metabolism: Revisiting the central dogma. Bioessays 36, 226-235.

de Lorenzo, V. y Danchin, A. (2008) Synthetic biology, discovering new worlds and new words. EMBO Rep 9, 822-827. 
Dueber, J.E., Wu, G.C., Malmirchegini, G.R., Moon, T.S., Petzold, C.J., Ullal, A.V., Prather, K.L. y Keasling, J.D. (2009) Synthetic protein scaffolds provide modular control over metabolic flux. Nature Biotech 27, 753-759.

Endy, D. (2005) Foundations for engineering biology. Nature 438, 449-453.

Gibson, D.G., Glass, J.I., Lartigue, C., Noskov, V.N., Chuang, R.-Y., Algire, M.A., Benders, G.A., Montague, M.G., Ma, L. y Moodie, M.M. (2010) Creation of a bacterial cell controlled by a chemically synthesized genome. Science 329, 52-56.

Huerta, S. (2006) Structural design in the work of Gaudi. Architech Scie Rev 49, 324-339. Jacob, F. (1977) Evolution and tinkering. Science 196, 1161-1166.

Kosuri, S., Goodman, D.B., Cambray, G., Mutalik, V.K., Gao, Y., Arkin, A.P., Endy, D. y Church, G.M. (2013) Composability of regulatory sequences controlling transcription y translation in Escherichia coli. Proc Natl Acad Sci USA 110, 1402414029.

Malyshev, D.A., Dhami, K., Lavergne, T., Chen, T., Dai, N., Foster, J.M., Corrêa, I.R. y Romesberg, F.E. (2014) A semi-synthetic organism with an expanded genetic alphabet. Nature 509, 385-388.

Milanesio, P., Arce-Rodriguez, A., Munoz, A., Calles, B. y de Lorenzo, V. (2011) Regulatory exaptation of the catabolite repression protein (Crp)-cAMP system in Pseudomonas putida. Env Microbiol 13, 324-339.

Monod, J. (1970) Le hasard et la nécessité. Essai sur la philosophie naturelle de la biologie moderne. Editions du Seuil, Paris.

Mutalik, V.K., Guimaraes, J.C., Cambray, G., Mai, Q.A., Christoffersen, M. J., Martin, L., Yu, A., Lam, C., Rodriguez, C., Bennett, G., Keasling, J.D., Endy, D. y Arkin, A.P. (2013) Quantitative estimation of activity y quality for collections of functional genetic elements. Nat Methods 10, 347-353.

Paddon, C.J. y Keasling, J.D. (2014) Semi-synthetic artemisinin, a model for the use of synthetic biology in pharmaceutical development. Nat Revs Microbiol 12, 355-367.

Porcar, M., Latorre, A. y Moya, A. (2013) What symbionts teach us about modularity. Front Bioeng Biotech 1.

Posfai, G., Plunkett, G., 3rd, Feher, T., Frisch, D., Keil, G.M., Umenhoffer, K., Kolisnychenko, V., Stahl, B., Sharma, S.S., de Arruda, M., Burland, V., Harcum, S.W. y Blattner, F.R. (2006) Emergent properties of reduced-genome Escherichia coli. Science 312, 1044-1046.

Schmidt, M. y de Lorenzo, V. (2012) Synthetic constructs in/for the environment, managing the interplay between natural y engineered Biology. FEBS Lett 586, 2199-2206.

Schrödinger, E. (1944) What is Life? Cambridge University Press, Cambridge.

Silva-Rocha, R. y de Lorenzo, V. (2008) Mining logic gates in prokaryotic transcriptional regulation networks. FEBS Lett 582, 1237-1244.

van Ham, R.C.H.J., Kamerbeek, J., Palacios, C., Rausell, C., Abascal, F., Bastolla, U., Fernández, J.M., Jiménez, L., Postigo, M., Silva, F.J., Tamames, J., Viguera, E., Latorre, A., Valencia, A., Morán, F. y Moya, A. (2003) Reductive genome evolution in Buchnera aphidicola. Proc Natl Acad Sci USA 100, 581-586.

Watson, J.D. (1968) The Double helix, A personal account of the discovery of the structure of DNA. Atheneum, New York. 